\title{
Spontaneous Resolution of a Choroidal Mass: A Case Series
}

\author{
Juan I. Bianchi ${ }^{a}$ Rajinder S. Nirwan ${ }^{b}$ Emiliano Fulda Graue ${ }^{a}$ \\ Adam Ross-Hirsch $^{b}$ Salvador Lopez-Rubio ${ }^{a}$ David A. DiLoreto Jr. ${ }^{b}$ \\ Arun D. Singh ${ }^{c}$
}

a Ophthalmology Institute, Fundación Conde de Valenciana, Mexico City, Mexico; ${ }^{b}$ Flaum Eye Institute, University of

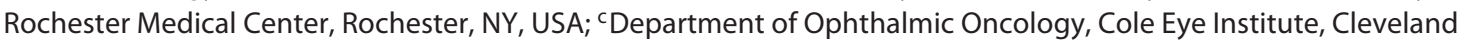
Clinic, Cleveland, OH, USA

\section{Established Facts}

- Choroidal masses can be of varied etiology.

- Multimodal imaging techniques including standardized A-scan and B-scan ultrasonography, fluorescein angiography, indocyanine green angiography, and optical coherence tomography are essential.

- In appropriate cases, systemic investigations may also be required.

\section{Novel Insights}

- Choroidal masses (non-neoplastic) can resolve spontaneously.

- Despite the use of multimodal imaging techniques, the etiology of choroidal masses may remain elusive.

- Extensive systemic investigations may have to be repeated to identify their underlying etiology.

\section{Keywords}

Choroidal mass · Metastasis · Inflammation · Spontaneous resolution

\section{Abstract \\ Choroidal masses can be of varying etiologies including tu- mors of benign, primary, and metastatic nature. Herein, we re- port on 3 cases of well-documented solitary choroidal masses associated with exudative retinal detachments of unclear etiol- ogy (despite extensive workup) that resolved spontaneously.}

(c) 2020 S. Karger AG, Basel

\section{Introduction}

Choroidal masses that produce exudative retinal detachments can be of varying etiologies including tumors of benign, primary malignant, and metastatic nature [1]. Furthermore, infectious or noninfectious inflammatory disorders, such as sarcoidosis, may also simulate a uveal tumor [2]. Finally, choroidal effusion or hemorrhage should also be considered in the differential diagnosis of a choroidal mass.

In addition to ophthalmoscopic examination, ancillary studies such as angiography (fluorescein or indocya-

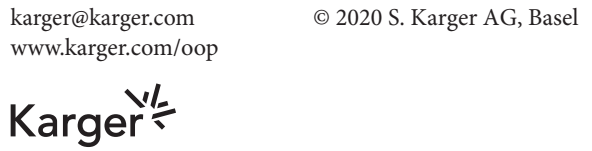

Arun D. Singh

Department of Ophthalmic Oncology

Cole Eye Institute, Cleveland Clinic

9500 Euclid Avenue, Desk i32, Cleveland, OH 44195 (USA)

singha@ccf.org 

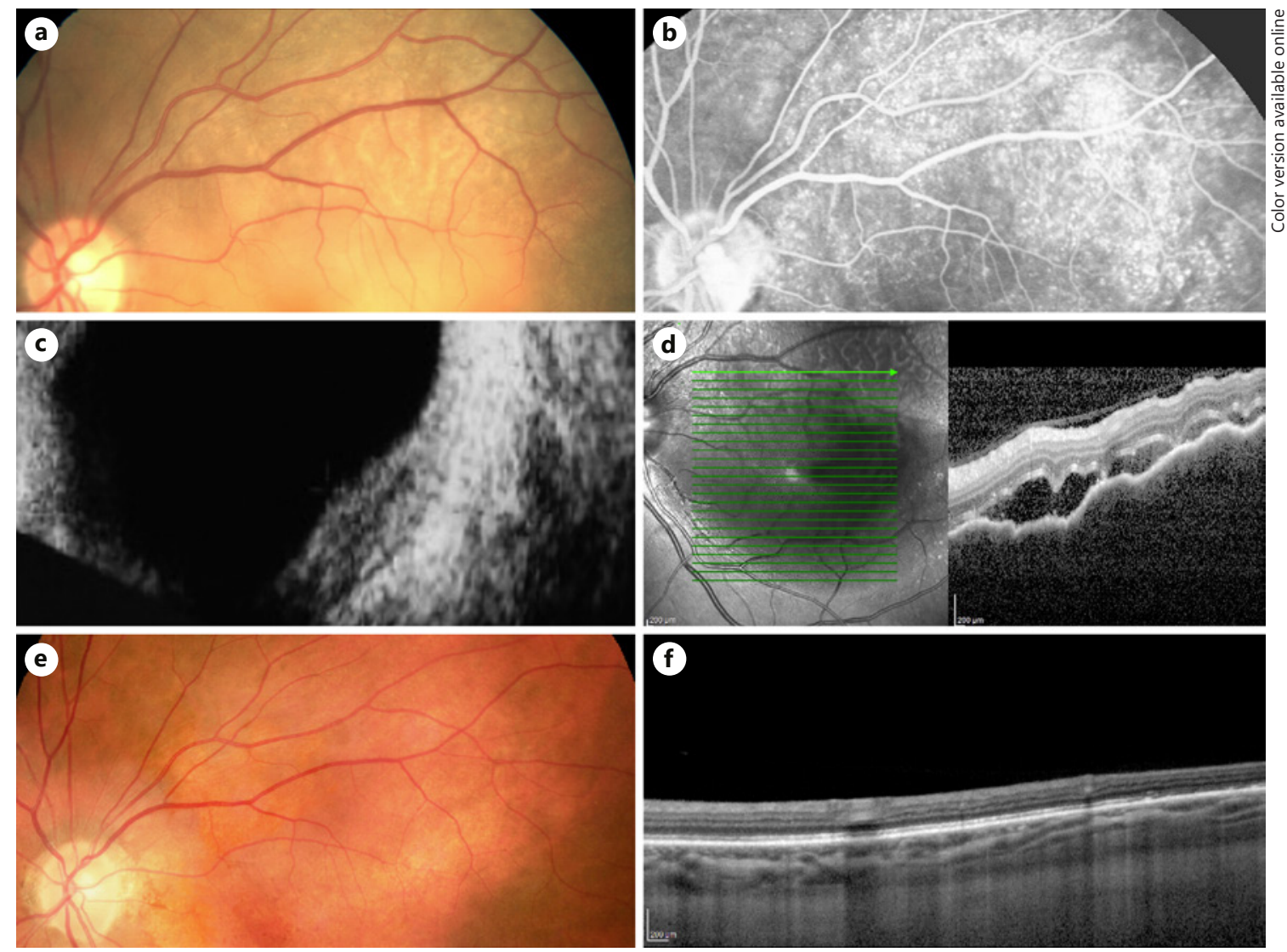

Fig. 1. a Left fundus photo at baseline showing an orange-yellow choroidal mass with poorly defined edges and overlying pigment mottling. The associated subretinal fluid (SRF) involved the fovea. b Note the multiple hyperfluorescent pinpoint leakages in the late phases of the fluorescein angiography. c Ultrasonography image confirming the dome-shaped choroidal mass with heterogeneous composition and medium-to-high internal reflectivity. d Wavy al-

nine green) [3], ultrasonography (USG; standardized A scan) [4], and optical coherence tomography (OCT) can aid in making a correct diagnosis [5]. In an atypical presentation, diagnostic fine needle aspiration biopsy (FNAB), or even incisional/excisional biopsy, may be required if the systemic assessment for inflammatory disorders (serology) or malignancy (imaging) is unrevealing [6].

In general, there is an urgent need for a correct diagnosis due to the concern for irreversible vision loss and progression of the underlying process (e.g., metastases). Herein, we report on 3 cases of well-documented solitary choroidal masses associated with exudative retinal detachments of unclear etiology (despite extensive workup) that resolved spontaneously. teration of the retinal pigment epithelium and choroidal infiltration on enhanced depth imaging optical coherence tomography (EDI-OCT). e Left fundus photo 9 months following initial presentation, demonstrating a residual flat, orange choroidal discoloration and absence of SRF. $\mathbf{f}$ At the final visit, EDI-OCT confirmed a normal retinal architecture, absence of SRF, and a choroid without alterations.

\section{Case Descriptions}

Case 1

A 60-year-old female, with no significant past medical history, presented with sudden onset of painless vision loss in the left eye. The best corrected visual acuity (BCVA) was 20/20 in the right eye and 20/400 in the left eye. The intraocular pressure (IOP) was 16 $\mathrm{mm} \mathrm{Hg}$, with normal anterior segment examination findings for both eyes. Fundus examination of the right eye was normal. In the left eye, the optic disc was normal. However, in the region of the superotemporal arcade, an orange-yellow subretinal mass $(14 \times$ $10 \times 3.5 \mathrm{~mm}$ ) with poorly defined edges was observed.

Overlying pigment mottling with subretinal fluid (SRF), which extended to the subfoveal area, was noted (Fig. 1a). Fluorescein angiography showed early hypofluorescence followed by multiple pinpoint areas of leakage in the later phases (Fig. 1b). Indocyanine green angiography demonstrated early hypofluorescence of the lesion with late staining. USG showed a dome-shaped choroidal 


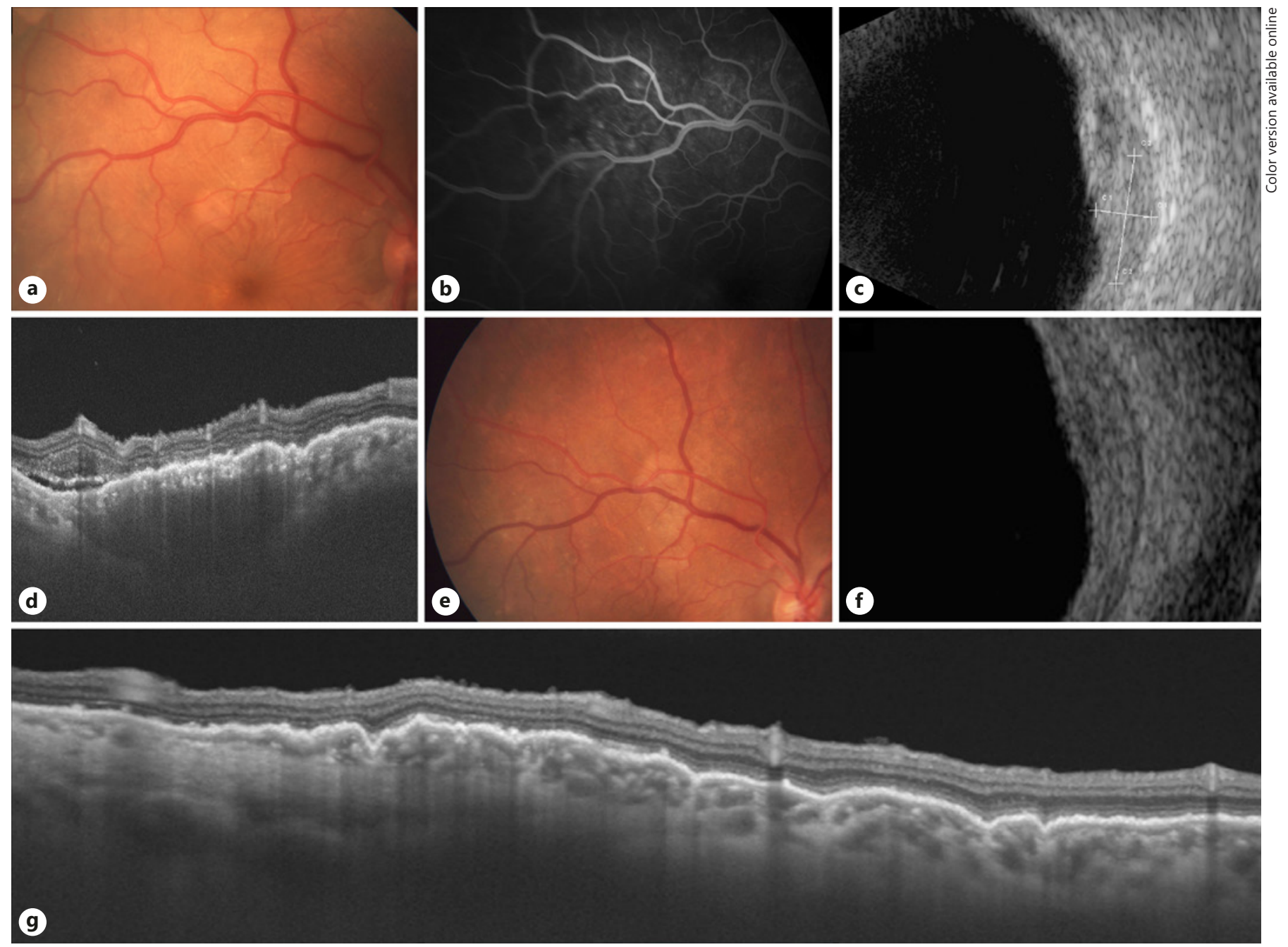

Fig. 2. a Color fundus photo of the right eye displaying an ill-defined yellow choroidal mass centered at the superior vascular arcade with surrounding subretinal fluid (SRF) that extends to the macula. b Multiple hyperfluorescent pinpoint areas of leakage on fluorescein angiography. c Ultrasonography (USG) revealing a dome-shaped choroidal lesion with medium internal reflectivity and surrounding SRF. d Swept-source optical coherence tomography (SS-OCT) showing SRF and a wavy alteration of the retinal pigment epithelium (RPE). Hyperreflective dots were noted within the superficial choroid and the inner retina. e Color fundus photo 6 weeks following initial presentation with residual retinal folds, ill-defined hypopigmented choroidal elevation, and absence of SRF. f USG confirmed a residual choroidal mass with a normal retinal architecture, and absence of SRF. $\mathbf{g}$ SS-OCT performed after 10 months demonstrated resolution of the choroidal mass, although the wavy alteration of the RPE remained. mass with a heterogeneous composition and medium-to-high internal reflectivity (Fig. 1c). Enhanced depth imaging OCT displayed choroidal infiltration and wavy alteration of the retinal pigment epithelium (RPE). Hyperreflective dots were noted within the SRF and in the inner retina (Fig. 1d). A computed tomography scan of the chest, abdomen and pelvis, magnetic resonance imaging of the brain, and a blood count with serum protein electrophoresis were negative.

One week later, the patient reported spontaneous visual improvement (BCVA of 20/80) corresponding to a reduction of SRF on examination. The patient was monitored periodically with progressive improvement. Nine months following the initial presenta- tion, the BCVA was 20/25 with a residual flat area of orange choroidal discoloration $(7.5 \times 6 \times 1.0 \mathrm{~mm})$ and absence of SRF (Fig. 1e). Enhanced depth imaging OCT confirmed a normal retinal architecture, absence of SRF, and a normal choroid (Fig. 1f).

\section{Case 2}

A 65-year-old female presented 9 days following uncomplicated cataract surgery of the right eye with the chief complaint of reduced vision in that eye. The BCVA of the right eye was 20/50 (+2.75 $-2.25 \times 70$ ). Her past medical history was noncontributory. Examination revealed mild corneal edema, anterior chamber cells, a posterior chamber intraocular lens in the capsular bag, and an IOP of 

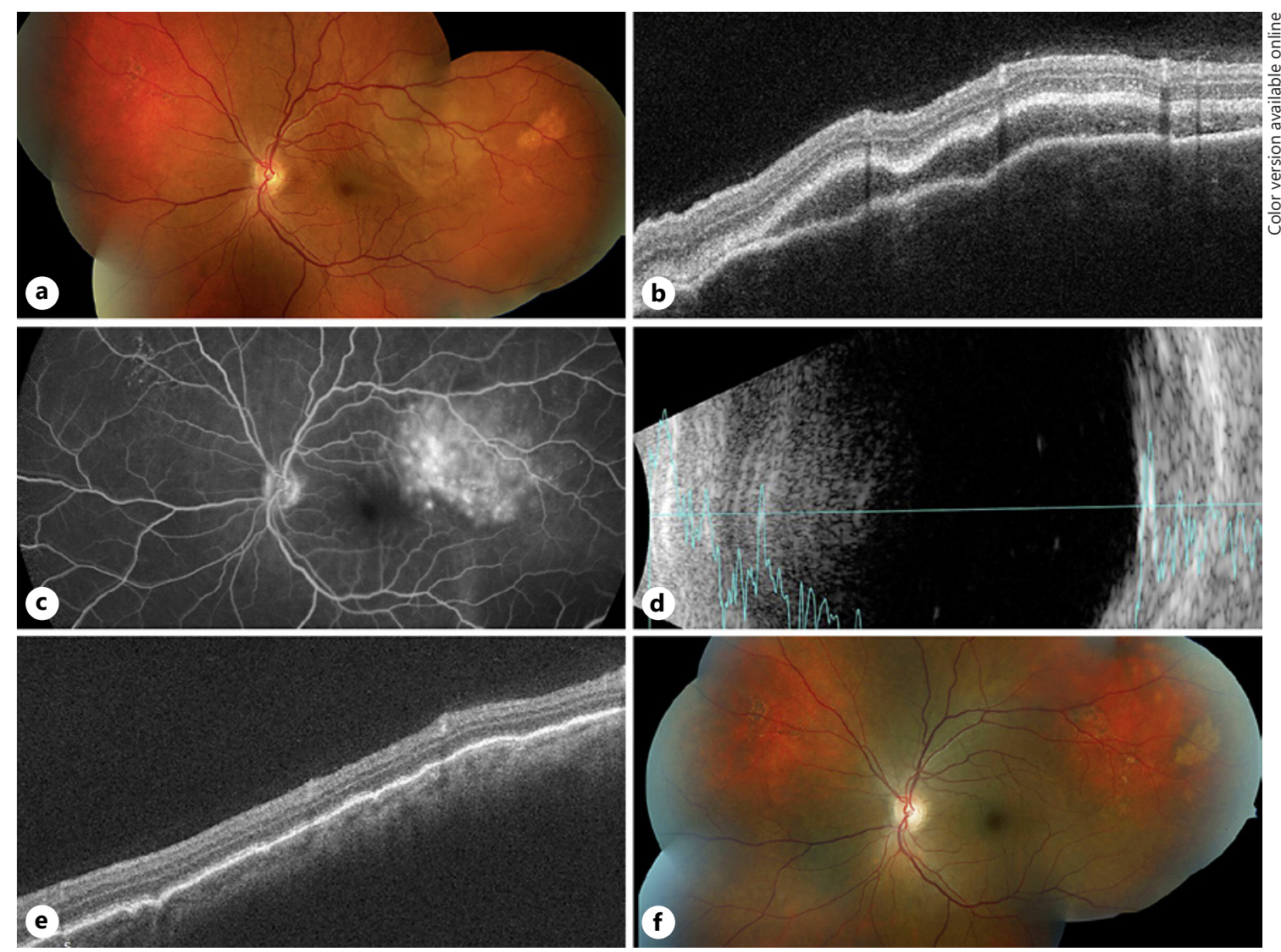

Fig. 3. a Color fundus photo of the left eye demonstrating an orange choroidal mass near the superotemporal arcade with subretinal fluid (SRF) and retinal folds extending towards the fovea. b Optical coherence tomography (OCT) of the left eye illustrating an irregular choroidal contour with SRF. c Multiple areas of late leakage on fluorescein angiography of the left eye. d Ultrasonography of the left eye showing an elevated, acoustically solid mass with high internal reflectivity. e Complete resolution of SRF on OCT of the left eye 5 weeks after the first injection of bevacizumab; however, the wavy, irregular contour of the retinal pigment epithelium remained. f Color fundus photo of the left eye 15 months after initial presentation with hyperpigmentation, areas of atrophy, and resolution of SRF.
$12 \mathrm{~mm} \mathrm{Hg}$. Fundus examination was limited but was grossly normal. Although the anterior segment inflammation improved with 1 week of therapy with topical steroids (prednisolone), antibiotics (moxifloxacin), and anti-inflammatory agents (nepafenac ophthalmic suspension), the BCVA worsened to $20 / 100(+3.25-0.85 \times 65)$.

Fundus examination at this visit revealed SRF in the macula with an ill-defined yellow-colored chorioretinal lesion that was centered at the superior vascular arcade (Fig. 2a). Fluorescein angiography exhibited early hypofluorescence followed by multiple hyperfluorescent pinpoint areas of leakage (Fig. 2b). USG confirmed a dome-shaped choroidal lesion with medium internal reflectivity and surrounding SRF (Fig. 2c). Choroidal infiltration and wavy alteration of the RPE were observed on OCT. Hyperreflective dots were noted within the superficial choroid and in the inner retina (Fig. 2d). A computed tomography scan of the chest, abdomen and pelvis, magnetic resonance imaging of the brain, and a blood count with serum protein electrophoresis were negative.

The patient was monitored periodically, with progressive spontaneous improvement. Six weeks following initial presentation, the
BCVA was $20 / 20$ in the right eye $(+0.50-1.25 \times 85)$ with a normal anterior segment. Residual retinal folds were present, with ill-defined hypopigmented choroidal elevation and absence of SRF (Fig. 2e). USG confirmed a residual choroidal mass with a normal retinal architecture, as well as absence of SRF (Fig. 2f). Ten months after initial presentation, the BCVA remained 20/20 with a flat, granular, hypopigmented area. Only a wavy alteration of the RPE and a minimal choroidal mass were detected on swept-source OCT (Fig. 2g).

\section{Case 3}

A 55-year-old African-American woman with a past medical history significant for hypertension and hyperthyroidism presented for urgent evaluation of increasing blurry vision in the right eye over a period of 12 weeks. She had mild pain and floaters in the right eye. Her BCVA was 20/20 and 20/60 in the right and the left eye, respectively. Her pupillary response, IOP, and extraocular eye movements were within normal limits.

Fundoscopic examination of her left eye demonstrated macular folds with SRF and a choroidal mass $(11 \times 9 \mathrm{~mm})$ in the supe- 
rotemporal arcade, extending to the center of the macula (Fig. 3a). OCT displayed an irregular choroidal contour and confirmed the presence of SRF (Fig. 3b). Fluorescein angiography was performed and displayed early hypofluorescence interspersed with hyperfluorescence that gradually leaked over time (Fig. 3c). USG revealed an elevated, acoustically solid mass with high internal reflectivity (Fig. 3d). A pertinent system review was negative. Further workup, including imaging and serology studies, was performed to rule out other possible diagnoses, all of which was negative. Five weeks following a single intravitreal injection of bevacizumab $(1.25 \mathrm{mg}$ in $0.05 \mathrm{~mL}$ ), her BCVA improved to $20 / 25$ with complete resolution of the SRF as well as a decrease in the size of the choroidal mass (Fig. 3e). Over a 15-month period, the patient received a total of 4 intravitreal injections without recurrence of SRF. At the most recent visit, the BCVA was stable at 20/25, and the fundus demonstrated a flat, pigmented area with atrophy (Fig. 3f). The mass decreased in size after the first injection and progressively resolved.

\section{Discussion}

Spontaneous regression of choroidal tumors such as hemangiomas $[7,8]$ or melanomas is extremely uncommon [9-13]. To the best of our knowledge, there are only 2 reports of metastases from renal carcinomas that have undergone spontaneous resolution after nephrectomy $[14,15]$. Other entities such as posterior scleritis, choroidal hemorrhage, or varix of the vortex vein ampulla can mimic an intraocular mass and may resolve spontaneously [16]. Spontaneous resolution can be expected if the choroidal mass is secondary to localized hemorrhage [2]. Only a few cases of choroidal inflammation that have undergone spontaneous resolution have been reported $[17,18]$.

Our 3 cases reported herein appear to have a set of unique clinical features. The most impressive feature is that of spontaneous resolution of the choroidal mass and associated exudative retinal detachment. The ophthalmic history, ocular examination, and ophthalmologic workup allow to rule out certain diagnoses such as posterior scleritis, choroidal hemorrhage, and varix of the vortex vein ampulla. Choroidal tumors like melanoma, lymphoma, and hemangioma could also be excluded [2].

Nevertheless, no definitive etiology could be established in any of the cases despite extensive radiologic and serologic investigations. Our main concern was to rule out choroidal metastatic tumors. The wavy appearance of the RPE anterior to the tumor, as seen on OCT in our patients, was similar to the irregular and "lumpybumpy" contour reported with choroidal metastasis [19]. FNAB is a relatively safe and effective diagnostic procedure, especially when a definitive diagnosis cannot be achieved despite ancillary studies and systemic investigations $[6,20]$. Cytologic evaluation of the FNAB sample in 1 published case had revealed nonspecific inflammation, excluding a neoplastic process [18]. The immediate onset in our case 1 after an intraocular surgery, and the presence of hyperreflective dots in the retina, subretinal space, and choroid on OCT in case 2 , suggest a probable inflammatory etiology for both.

Inflammation manifesting as a choroidal granuloma or nodular scleritis can mimic amelanotic choroidal melanoma and other intraocular tumors [21, 22]. Common causes include sarcoidosis, tuberculosis, and nontuberculous mycobacterial infections [23-25]. Systemic investigations are necessary to establish the underlying cause.

Spontaneous improvement was observed in these cases while the systemic investigations were being undertaken. In the presence of spontaneous improvement and unrevealing systemic investigations, we elected to continue the observation, which eventually led to the total resolution of the masses. None of the cases received any systemic therapy such as antibiotics or steroids that could have reversed the inflammation or infection. Over the average follow-up period of 10 months (range 6-15), there has been no local recurrence or onset of systemic signs or symptoms suggestive of systemic inflammatory disorder or underlying malignant tumor. In summary, our 3 cases likely represent unusual forms of choroidal inflammation of undetermined etiology that resolved spontaneously without delayed recurrence.

\section{Statement of Ethics}

Since this is a retrospective case study that included deidentified descriptions, it was exempt from Institutional Review Board approval. The patient in the USA provided written informed consent for publication, and the 2 patients in Mexico City provided oral informed consent for their clinical images to be used for scholarly purposes including scientific publications. While we do not have personalized written informed consent for clinical cases, patients know that images of their eyes (all types of image as long as the patient is not identified) can be used for scholarly purposes including scientific publications. Patients are aware of this and agree to this policy. The images are anonymized.

\section{Conflict of Interest Statement}

The authors have no conflicts of interest to declare. 


\section{Funding Sources}

No funding was received.

\section{References}

1 Shields JA, Mashayekhi A, Ra S, Shields CL. Pseudomelanomas of the posterior uveal tract: the 2006 Taylor R. Smith Lecture. Retina. 2005 Sep;25(6):767-71.

2 Char DH. Uveal melanoma: differential diagnosis. In: Damato BE, Singh AD, editors. Clinical ophthalmic oncology: uveal tumors. Heidelberg: Springer; 2014. Chapter 8, p. 99113.

3 Shields CL, Shields JA, De Potter P. Patterns of indocyanine green videoangiography of choroidal tumours. Br J Ophthalmol. 1995 Mar;79(3):237-45.

4 Turell ME, Hayden BC, Schoenfield LR, Singh AD. Intraocular tumors. In: Hayden BC, Singh AD, editors. Ophthalmic ultrasonography. Philadelphia (PA): Saunders- Elsevier; 2012. p. 111-31.

5 COMS Group. Accuracy of diagnosis of choroidal melanomas in the Collaborative Ocular Melanoma Study. COMS report No. 1. Arch Ophthalmol. 1990 Sep;108(9):1268-73.

6 Singh AD, Pelayes DE, Brainard JA, Biscotti CV. History, indications, techniques and limitations. Monogr Clin Cytol. 2012;21:1-9.

7 Pitta C, Bergen R, Littwin S. Spontaneous regression of a choroidal hemangioma following pregnancy. Ann Ophthalmol. 1979 May; 11(5):772-4.

8 Sayman Muslubas I, Arf S, Hocaoglu M, Ozdemir H, Karacorlu M. Spontaneous Regression of Serous Retinal Detachment Associated with Circumscribed Choroidal Hemangioma after Childbirth. Retin Cases Brief Rep. 2017;11(1):7-11.

\section{Author Contributions}

All authors contributed to the diagnosis, as well as to the management of the described patients. All authors also contributed to the collection of data and to writing and/or editing of the manuscript.

9 Davidorf FH. Spontaneous regression of a choroidal melanoma? Arch Ophthalmol. 1986 Sep;104(9):1272-3.

10 Lambert SR, Char DH, Howes E Jr, Crawford JB, Wells J. Spontaneous regression of a choroidal melanoma. Arch Ophthalmol. 1986 May; 104(5):732-4.

11 Chong CA, Gregor RJ, Augsburger JJ, Montana J. Spontaneous regression of choroidal melanoma over 8 years. Retina. 1989;9(2): 136-8.

12 Shields CL, Shields JA, Santos MC, Gündüz K, Singh AD, Othmane I. Incomplete spontaneous regression of choroidal melanoma associated with inflammation. Arch Ophthalmol. 1999 Sep;117(9):1245-7.

13 Jain A, Gupta M, Rundle PA, Rennie IG. Spontaneous regression of choroidal melanoma. Eye (Lond). 2006 Aug;20(8):959-61.

14 Langmann G, Müllner K. Spontaneous regression of a choroidal metastasis from renal carcinoma. Br J Ophthalmol. 1994 Nov; 78(11):883.

15 Hammad AM, Paris GR, van Heuven WA, Thompson IM Jr, Fitzsimmons TD. Spontaneous regression of choroidal metastasis from renal cell carcinoma. Am J Ophthalmol. 2003 Jun;135(6):911-3.

16 Shields CL, Pellegrini M, Kligman BE, Bianciotto C, Shields JA. Ciliary body and choroidal pseudomelanoma from ultrasonographic imaging of hypermature cataract in 20 cases. Ophthalmology. 2013 Dec;120(12):2546-51.
17 Gupta M, Singh AD, Stavrou P, Rundle PA, Rennie IG. Spontaneous resolution of a choroidal mass. Eye (Lond). 2005 May; 19(5): 601-3.

18 Bianchi JI, Graue EF, Lopez-Rubio S, Bellerive C, Biscotti CV, Singh AD. Diagnostic and therapeutic challenges. Retina. 2019 Aug; 39(8):1632-4.

19 Al-Dahmash SA, Shields CL, Kaliki S, Johnson T, Shields JA. Enhanced depth imaging optical coherence tomography of choroidal metastasis in 14 eyes. Retina. 2014 Aug;34(8): 1588-93.

20 Bellerive C, Biscotti CV, Singh N, Singh AD. Fine-needle aspiration biopsy for suspected uveal metastases. Can J Ophthalmol. 2019 Dec;54(6):694-8.

21 Alsharif HM, Al-Dahmash SA. Atypical posterior scleritis mimicking choroidal melanoma. Saudi Med J. 2018 May;39(5):514-8.

22 Welch RJ, Newman JH, Honig SE, Mayro EL, McGarrey M, Graf AE, et al. Choroidal amelanotic tumours: clinical differentiation of benign from malignant lesions in 5,586 cases. $\mathrm{Br}$ J Ophthalmol. 2020 Feb;104(2):194-201.

23 Salah S, Abad S, Monnet D, Brézin AP. Sarcoidosis. J Fr Ophtalmol. 2018 Dec; 41(10):e451-67.

24 Sassalos TM, Rao RC, Demirci H. Ocular tuberculosis masquerading as a tumor. Lancet Infect Dis. 2018 Aug;18(8):924.

25 Moorthy RS, Valluri S, Rao NA. Nontuberculous mycobacterial ocular and adnexal infections. Surv Ophthalmol. 2012 May-Jun;57(3): 202-35. 